\title{
A RESULT ON CO-CHROMATIC GRAPHS
}

\author{
E.J. FARRELL \\ Department of Mathematics \\ The University of the West Indies \\ St. Augustine, Trinidad. \\ (Received December 17, 1979 and in revised \\ form Aprf1 18, 1980)
}

ABSTRACT. A sufficient condition for two graphs with the same number of nodes to have the same chromatic polynomial is given.

KEY WORDS AND PHRASES. Graph, Co-chromatic, Chromatic polynomial. 1980 MATHEMATICS SUBJECT CLASSIFICATION CODES: Primary 05 C15, Secondary 26 C10.

1. INTRODUCTION.

We prove a theorem which gives a sufficient condition for two graphs to be co-chromatic 1.e. to have the same chromatic polynomial.

The chromatic polynomial $\chi(G ; \lambda)$ of a graph $G$ with $p$ nodes has degree $p$ and constant term equal to 0 . Hence the chromatic polynomial has p coefficients. If the graph has at least one edge, then the sum of these coefficients is equal to 0 . Hence the chromatic polynomial is uniquely determined if p-1 of the coeffictents are known. Our result is a generalization of this.

2. MAIN RESULTS. 
THEOREM 2. If two graphs with $\mathrm{p}$ nodes have chromatic numbers $\geq \mathrm{n}$ and have at least $p+1-n$ corresponding coefficients of their chromatic polynomials equal, then they are co-chromatic.

In the proof we shall use a spectal case of the following Lemma.

LEMMA 2.1

Let $P(x)=c_{1} x^{n_{1}}+c_{2} x^{n_{2}}+\ldots+c_{s} x^{n_{s}}$, where $c_{1}$ and $n_{1}$ are real numbers for $1=1$, $2, \ldots, s$. We assume that $c_{i} \neq 0$ for all 1 and that $n_{i} \neq n_{j}$ for $i \neq j$. Then the equation $P(x)=0$ has at most $s-1$ real positive solutions.

PROOF OF THE LEMMA 2.1 By induction over $s$. For $s=1$ the statment is obvious. Suppose that it is true for $s-1$ and let $P(x)$ be the above expression. The expression

$$
Q(x)=x^{-n_{1}} \cdot P(x)=c_{1}+c_{2} x^{n_{2}-n_{1}}+\ldots c_{s} x^{n_{s}-n_{1}}
$$

has the derivative

$$
Q^{\prime}(x)=c_{2}\left(n_{2}-n_{1}\right) \cdot x^{n_{2}-n_{1}-1}+\ldots+c_{s}\left(n_{s}-n_{1}\right) \cdot x^{n_{s}-n_{1}-1} .
$$

By induction, $Q^{\prime}(x)=0$ for at most $s-2$ positive $x$. But between any two positive solutions of $P(x)=x^{n_{1}} \cdot Q(x)=0$, there exists a solution of $Q^{\prime}(x)=0$. Hence $P(x)=0$ for at most $s-1$ positive $x$.

$$
\text { Q.E.D. }
$$

PROOF OF THE THEOREM 2. Let $G$ and $H$ be the two graphs. Let us assume that $m$ of the coefficients of $\chi(G ; \lambda)$ and $\chi(H ; \lambda)$ are equal. Then $m \geq p+1-n$, by our assumption. Let us assume that $m<p$. We can therefore write

and

$$
\chi(G ; \lambda)=f(\lambda)+g(\lambda)
$$$$
x(H ; \lambda)=f(\lambda)+h(\lambda),
$$

where $f(\lambda)$ contains $m$ terms and $g(\lambda)$ and $h(\lambda)$ are the remaining terms of $\chi(G ; \lambda)$ and $X(H ; \lambda)$ respectively.

Since $G$ and $H$ have chromatic numbers $\geq n$, all integers $1,2, \ldots, n-1$ are roots of $X(G ; \lambda)$ and $X(H ; \lambda)$. 
Let

$$
g(\lambda)=a_{1} \lambda^{n_{1}}+a_{2} \lambda^{n_{2}}+\ldots+a_{p-m} \lambda^{n_{p-m}}
$$

and $\quad h(\lambda)=b_{1} \lambda^{n_{1}}+b_{2} \lambda^{n_{2}}+\ldots+b_{p-m} \lambda^{n_{p-m}}$

If $r$ is an integer such that $1 \leq r \leq n-1$, then $g(r)=h(r)$, i.e.

$$
\left(a_{1}-b_{1}\right) r^{n_{1}}+\left(a_{2}-b_{2}\right) r^{n_{2}}+\ldots+\left(a_{p-m}-b_{p-m}\right) r^{n_{p-m}}=0 .
$$

Since $\mathrm{n}-1 \geq \mathrm{p}-\mathrm{m}$, this is a contradiction by the Lemma.

Q.E.D.

\section{ILLUSTRATIONS}

We will now 1llustrate the theorem. We will assume that the chromatic polynomial of a graph $G$ with $p$ nodes is written in descending powers of $\lambda$.

$$
\text { 1.e. } x(G ; \lambda)={ }_{k} \sum_{0} a_{p-k} \lambda^{p-k} \text {. }
$$

It is well known that if $G$ contains $p$ nodes and $q$ edges, then $a_{p}, a_{p-1}$ and $a_{p-2}$ are $1,-q$ and $\left({ }_{2}^{q}\right)-A$ respectively, where $A$ is the number of triangles in G. It was also shown in Farre11 [1] (Theorem 1) that

$$
a_{p-2}=-\left(\begin{array}{l}
q \\
3
\end{array}\right)+(q-2) A+B-2 C,
$$

where $B$ and $C$ are the numbers of subgraphs of $G$ which are quadrilaterals (without diagonals) and complete graphs with four nodes.

Let $G_{1}$ and $G_{2}$ be the graphs shown below

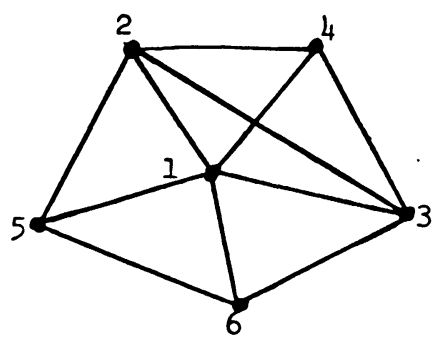

$\mathbf{G}_{1}$

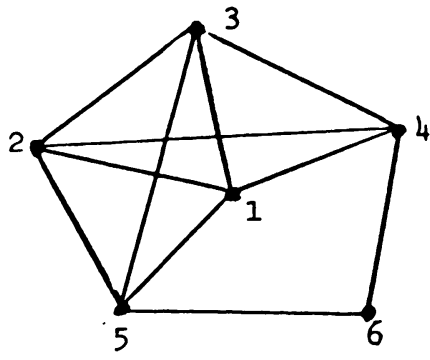

$\mathrm{G}_{2}$ 
Let $x\left(G_{1} ; \lambda\right)={ }_{k} \underline{\Sigma}_{0} a_{6-k} \lambda^{6-k}$ and $x\left(G_{2} ; \lambda\right)={ }_{k} \underline{\Sigma}_{0} b_{6-k} \lambda^{6-k}$. Then $a_{6}=b_{6}=1$ and and $a_{5}=b_{5}=11$. Since $G_{1}$ and $G_{2}$ contain 7 triangles, $a_{4}=b_{4}=\left(\begin{array}{c}11 \\ 2\end{array}\right)-7=48$. Therefore $G_{1}$ and $G_{2}$ have 6 nodes, their chromatic number is $\geq 4=n$ and $(p+1-n)=3$ of their corresponding coefficients are equal. It follows from the above theorem that $G_{1}$ and $G_{2}$ are co-chromatic.

The chromatic polynomial of $G_{1}$ and $G_{2}$ has been computed. It is $\chi\left(G_{1} ; \lambda\right)=\chi\left(G_{2} ; \lambda\right)=\lambda^{6}-11 \lambda^{5}+48 \lambda^{4}-103 \lambda^{3}+107 \lambda^{2}-42 \lambda$.

Consider the graphs $\mathrm{H}_{1}, \mathrm{H}_{2}$ and $\mathrm{H}_{3}$ shown below.

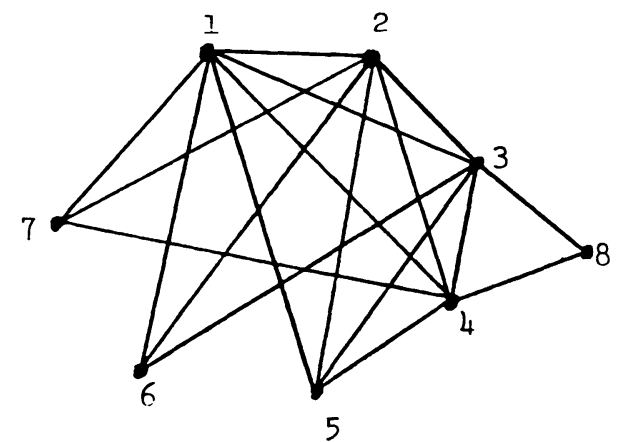

$\mathrm{H}_{1}$

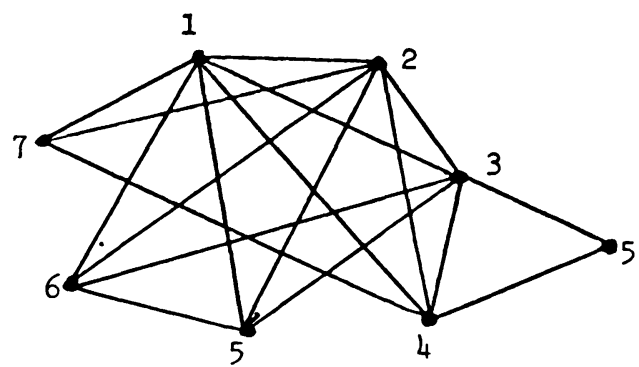

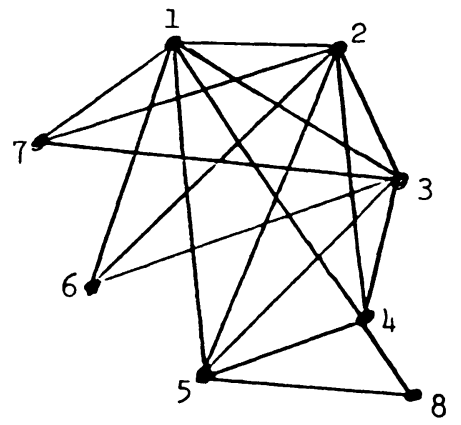

$\mathrm{H}_{2}$

$\mathrm{H}_{3}$

All three graphs contain 8 nodes and 18 edges. Each contains 17 triangles.

Therefore, the third coefficient of their chromatic polynomial is $\left(\begin{array}{c}18 \\ 2\end{array}\right)-17=136$.

Finally, each contains 7 subgraphs which are complete graphs with 4 nodes and

0 quadrilaterals without diagonals. Therefore the fourth coefficients are equal. 
Hence from the above theorem $\mathrm{H}_{1}, \mathrm{H}_{2}$ and $\mathrm{H}_{3}$ are co-chromatic.

The chromatic polynomial of $\mathrm{H}_{1}, \mathrm{H}_{2}$ and $\mathrm{H}_{3}$ has been computed. It is

$\chi\left(\mathrm{H}_{1} ; \lambda\right)=\chi\left(\mathrm{H}_{2} ; \lambda\right)=\chi\left(\mathrm{H}_{3} ; \lambda\right)=\lambda^{8}-18 \lambda^{7}+136 \lambda^{6}-558 \lambda^{5}+1339 \lambda^{4}-1872 \lambda^{3}+1404 \lambda^{2}$

$-432 \lambda$.

\section{REFERENCES}

[1] Farre11, E.J., On Chromatic Coefficients, Discrete Mathematics, 29(1980), 257-264. 


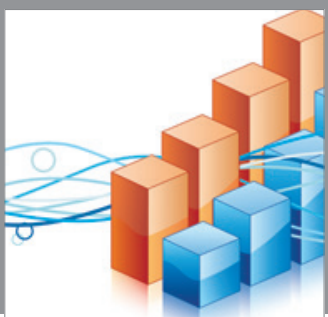

Advances in

Operations Research

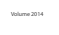

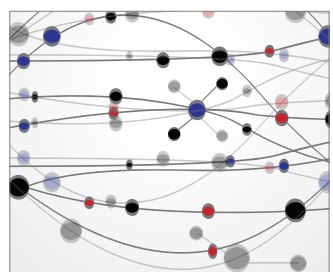

\section{The Scientific} World Journal
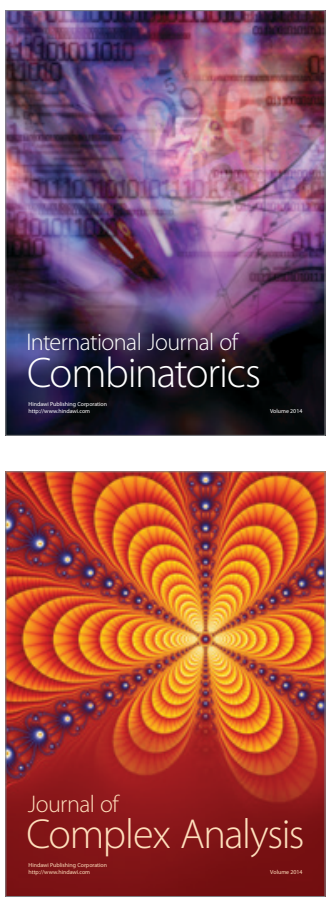

International Journal of

Mathematics and

Mathematical

Sciences
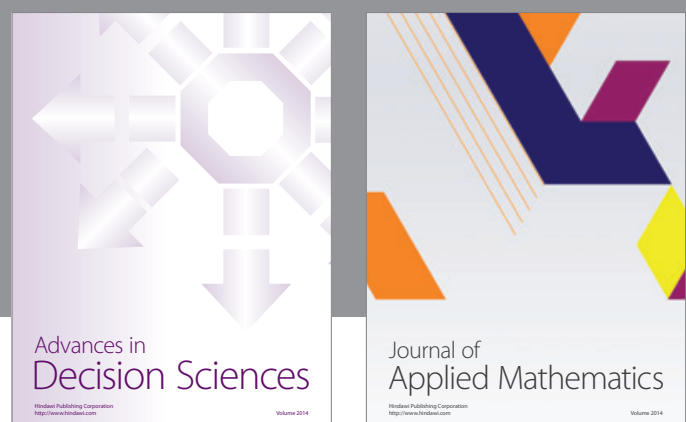

Journal of

Applied Mathematics
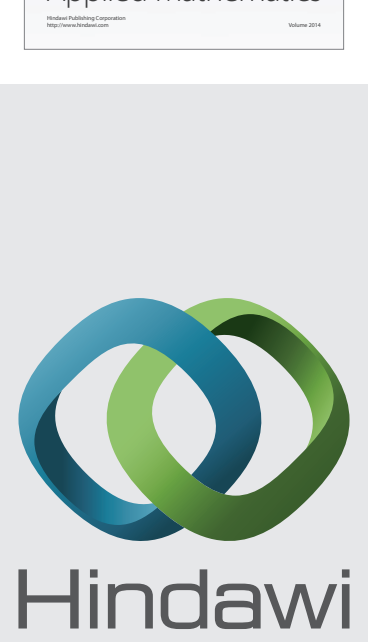

Submit your manuscripts at http://www.hindawi.com
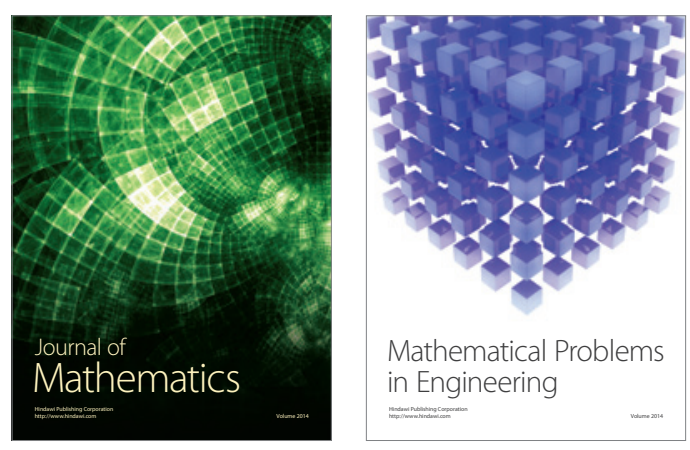

Mathematical Problems in Engineering
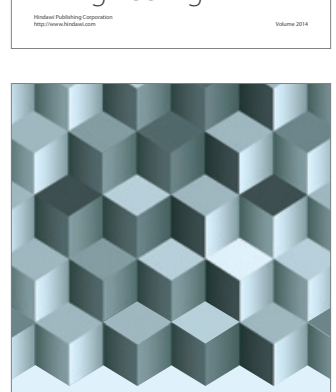

Journal of

Function Spaces
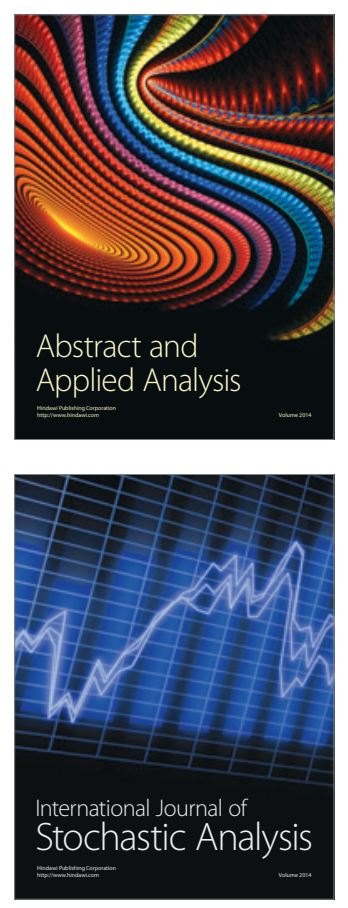

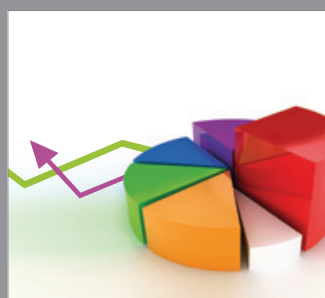

ournal of

Probability and Statistics

Promensencen
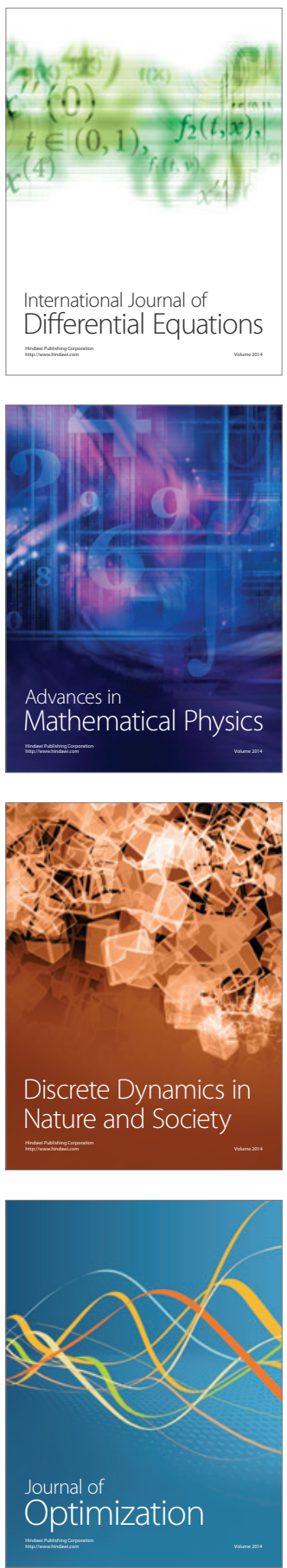